\title{
Associação entre periodontite crônica severa e reatividade microvascular cutânea de pacientes hipertensos
}

\author{
Raquel H. de Souza, ${ }^{1}$ Amanda Sant'Anna, ${ }^{1}$ Sérgio Kaiser, ${ }^{2}$ Márcia Simas, ${ }^{2}$ Ricardo G. Fischer ${ }^{1 *}$
}

\begin{abstract}
Resumo
Introdução: A periodontite (DP) é caracterizada como uma doença destrutiva que afeta os tecidos de sustentação dos dentes, podendo atingir gengiva, ligamento periodontal, cemento e osso alveolar. Objetivos: No presente estudo, 49 pacientes foram examinados para avaliar a possível associação da periodontite crônica grave na reatividade microvascular cutânea em pacientes hipertensos. Secundariamente, avaliou-se a relação da adiposidade corporal com a periodontite. Materiais e Métodos: O exame periodontal incluiu medidas de índice de placa e sangramento à sondagem, profundidade de bolsa à sondagem e nível de inserção clínica. A reatividade microvascular cutânea foi analisada por meio do Laser Speckle Contrast Imaging (LSCI), enquanto a adiposidade corporal foi examinada através da Bioimpedância elétrica (BIA). Resultados: Os resultados mostraram que não houve diferença na reatividade microvascular cutânea em pacientes com periodontite crônica grave em comparação com os pacientes periodontalmente saudáveis. Não foram observadas diferenças significantes entre hipertensos e normotensos. Com relação a adiposidade corporal, periodontite grave não influenciou os resultados, porém pacientes hipertensos demonstraram maiores índices de massa gorda em relação aos demais. Conclusões: A periodontite crônica severa não influenciou na reatividade microvascular cutânea em pacientes hipertensos e pessoas hipertensas apresentaram maior adiposidade corporal.
\end{abstract}

Descritores: Hipertensão; Obesidade; Periodontite; Bioimpedância; Laser Speckle.

\section{Abstract}

Association between severe chronic periodontitis and cutaneous microvascular reactivity in hypertensive patients

Introduction: Periodontitis (PD) is characterized as a destructive disease that affects the tissues that support the teeth, reaching gums, periodontal ligaments, cement and alveolar bone. Objectives: In the present study, 49 patients were examined to evaluate a possible association between severe chronic periodontitis on cutaneous microvascular reactivity in hypertensive patients. Secondarily, the relationship between body adiposity and periodontitis was also evaluated. Materials and Methods: The periodontal evaluation included measurements of plaque index and bleeding on probing, probing pocket depth and clinical attachment loss. Cutaneous microvascular reactivity was analyzed by means of the Laser Speckle Contrast Imaging (LSCI), while the body fatness was examined through Electric Bioimpedance (BIA). Results: The results showed no
1. Departamento Proclin. Faculdade de Odontologia. Universidade do Estado do Rio de Janeiro, Rio de Janeiro, RJ, Brasil

2. Clinex. Faculdade Ciências Médicas. Universidade do Estado do Rio de Janeiro, Rio de Janeiro, RJ, Brasil.

*Endereço para correspondência:

Faculdade de Odontologia, UERJ

Boulevard 28 de Setembro, 157

Rio de Janeiro, RJ, Brasil

CEP 22051-030

E-mail: ricfischer@globo.com

BJHBS, Rio de Janeiro, 2020;19(1):31-39

Received on 07/22/2019. Approved on 11/12/2020.

significant difference in cutaneous microvascular reactivity in patients with severe chronic periodontitis as compared with periodontally healthy patients. The were no significant differences between hypertensive and normotensive individuals. In relation to body adiposity, periodontitis did not influence the results, but hypertensive patients showed higher fat mass indexes as compared to non-hypertensive patients. Conclusion: In conclusion, severe chronic periodontitis did not influence cutaneous microvascular reactivity and hypertensive individuals had higher body adiposity.

Keywords: Hypertension; Obesity; Periodontal disease; Bioimpedance; Laser Speckle.

\section{Resumen}

Asociación entre periodontitis crónica grave y reactividad microvascular cutánea en pacientes hipertensos

Introdución: La periodontitis (EP) se caracteriza por ser una enfermedad destructiva que afecta los tejidos que sostienen los dientes, llegando a las encías, el ligamento periodontal, el cemento y el hueso alveolar. Objetivos: En el presente estudio, se examinaron 49 pacientes para evaluar la posible asociación de periodontitis crónica grave en la reactividad microvascular cutánea en pacientes hipertensos. En segundo lugar, se evaluó la relación entre la adiposidad corporal y la enfermedad periodontal. Materiales y Métodos: El examen periodontal incluyó mediciones del índice de placa y sangrado en el sondeo, profundidad de la bolsa en el sondeo y nivel de inserción clínica. La reactividad microvascular cutánea se analizó por medio de la imagen de contraste de moteado con láser (LSCI), mientras 


\section{Artigo original}

que la grasa corporal se examinó mediante bioimpedancia eléctrica (BIA). Resultados: Los resultados mostraron que no hubo diferencias en la reactividad microvascular cutánea en pacientes con periodontitis crónica grave en comparación con pacientes periodontalmente sanos. No hubo diferencias significativas entre los individuos hipertensos y normotensos. Con respecto a la adiposidad corporal, la periodontitis severa no influyó en los resultados, pero los pacientes hipertensos

\section{Introdução}

A Organização Mundial de Saúde (OMS) define doença comouma condiçãocausadora deconsequências adversas, incluindo prejuízos físicos ou psico $\neg$ lógicos, restrições de atividade e limitações de função. ${ }^{1}$ Nesse sentido, a periodontite (DP) é caracterizada como uma doença destrutiva que afeta os tecidos de sustentação dos dentes, podendo atingir gengiva, ligamento periodontal, cemento e osso alveolar. A DP caracteriza-se por processos imunoinflamatórios nos tecidos que circundam o dente, e compreende um processo interativo entre o biofilme dental e os tecidos periodontais. ${ }^{2}$ Quando a doença fica restrita aos tecidos gengivais e não envolve perda de inserção periodontal, ela é denominada gengivite, sendo a forma mais prevalente e branda da doença periodontal. ${ }^{3}$

Em termos epidemiológicos, 5 a 15\% da população é afetada pela periodontite crônica grave, enquanto a doença moderada atinge aproximadamente $50 \%$ dos adultos. ${ }^{4}$ A periodontite é resultado de uma complexa interação entre o desafio bacteriano e a resposta do hospedeiro, frequentemente modificada por fatores comportamentais. ${ }^{5}$ Entre esses fatores, a resposta do hospedeiro compreende o fator-chave para expressão clínica da periodontite, sendo o modificador mais importante para a gravidade da doença. ${ }^{6}$

O processo inflamatório crônico da periodontite e da resposta do hospedeiro fornecem a plausibilidade biológica para a associação entre periodontite e doença cardiovascular. Estudos sugerem que a pressão arterial sistólica e diastólica estão mais elevadas em indivíduos com periodontite em comparação com pacientes saudáveis. ${ }^{8-10} \mathrm{Em}$ um estudo com $12 \mathrm{mil}$ adultos dentados, nos Estados Unidos, foi demonstrada uma relação positiva entre pressão arterial sistólica e periodontite grave em pacientes de meia idade. ${ }^{9}$

Geralmente, os fatores de risco para doenças cardiovasculares (DCV) promovem o desenvolvimento de aterosclerose por meio de seus efeitos no endotélio vascular. ${ }^{11} \mathrm{O}$ endotélio normal não é aderente aos leucócitos circulantes. Porém, uma vez expostas mostraron índices de masa grasa más altos en relación con los demás. Conclusiónes: En conclusión, la periodontitis crónica grave no influyó en la reactividad microvascular cutánea en pacientes hipertensos y los individuos hipertensos presentaron mayor adiposidad corporal.

Palabras clave: Hipertensión; Obesidade; Enfermedad periodontal; Bioimpedancia; Speckle Laser.

a estímulos inflamatórios, as células endoteliais aumentam a expressão de moléculas de adesão como as moléculas de adesão intercelular-1 (ICAM-1), molécula de adesão celular vascular-1 (VCAM-1), P- selectinas e Beta 2 integrinas, permitindo então a inserção de leucócitos. ${ }^{12,13}$ Lira-Junior e colaboradores ${ }^{14}$ observaram uma associação entre disfunção microvascular e periodontite severa. Citocinas, produzidas pela periodontite, atuam no aumento da produção de vasoconstrictores no endotélio e promoção da adesão e agregação leucocitária, e podem predispor a trombogênese. ${ }^{15,16}$

As formas de avaliação da função endotelial são consideradas invasivas e não invasivas. Usualmente as formas invasivas são usadas para pacientes com comprometimento do endotélio comprovado e quando maior especificidade é requerida. Para a prática clínica usual de rotina e avaliação de pacientes saudáveis, métodos não invasivos mostram-se mais interessantes e contam com maior colaboração dos pacientes. Esses métodos incluem Dilatação mediada pelo fluxo (DMF), Fluxometria por laser Doppler (FLD), Pletismografia por oclusão venosa(POV) quando não há infusão de substâncias na artéria braquial e Velocidade de onda de pulso (VOP). Com amostras sanguíneas podem ser utilizadas as dosagens de substâncias circulantes secretadas pelo endotélio, nas quais se destacam fibrinogênio, fator de Von Willebrand, endotelina 1, moléculas de adesão e proteína $C$ reativa ultrassensível, podendo ser utilizadas como marcadores indiretos da ativação endotelial..$^{17}$ Especificamente para função endotelial coronariana, os testes não invasivos mais utilizados são o ecocardiograma com Doppler, a Tomografia com Emissão de Pósitrons (PET) e a ressonância nuclear magnética com contraste. ${ }^{18}$

O Laser Speckle Contrast Imaging (LSCI) é uma técnica não invasiva para avaliação da microcirculação cutânea com boa resolução temporal e espacial. OLSCI é usado para quantificar o fluxo sanguíneo basal a partir da pele, e ainda melhor, para avaliação da reatividade 
da microcirculação quando a pele é estimulada. Podem ser feitos estímulos mecânicos (oclusão da artéria ou pressão local) ou estímulos térmicos, e ainda o uso local de medicamentos. Portanto, o LSCI, quando acoplado a um teste de reatividade, é considerado de fácil execução e confiável para avaliar a microcirculação cutânea. ${ }^{19}$ Não existem relatos do uso do LSCI na avaliação da reatividade microvascular cutânea associada a periodontite.

Evidências indicam que uma dieta rica em carboidratos eleva os riscos de inflamação e sangramento gengival. ${ }^{20,21}$ Por outro lado, a dieta pobre em carboidratos resulta em menor sangramento gengival. ${ }^{21,22}$ Carboidratos fermentáveis têm sido apontados como os fatores de risco dietéticos mais comuns para cárie e doença periodontal. 21,23 Indivíduos obesos com maior número de dentes perdidos apresentam maior incidência de periodontite. ${ }^{24}$ Os carboidratos estão também relacionados à obesidade. O Índice de Massa Corporal (IMC) é o parâmetro mais utilizado para avaliar a presença de obesidade, porém apresenta limitações, podendo classificar erroneamente alguns indivíduos como obesos. ${ }^{25,26} \mathrm{Um}$ método mais apropiado para avaliação da adiposidade corporal é Bioimpedância Elétrica (BIA), pois estima a massa gorda. ${ }^{27}$ Não há estudos publicados avaliando a associação da periodontite com a obesidade avaliada por meio da BIA.

Dessa forma, o objetivo do presente estudo foi avaliar a associação entre periodontite crônica severa e a reatividade microvascular cutânea em pacientes hipertensos. Um objetivo secundário foi avaliar a associação da adiposidade corporal com periodontite crônica grave.

\section{Materiais e métodos}

O presente estudo foi realizado no laboratório da Disciplina de Fisiopatologia Clínica e Experimental (CLINEX) da Universidade do Estado do Rio de Janeiro (UERJ), e aprovado pelo Comité de Ética em Pesquisa do Hospital Universitário Pedro Ernesto (Registro CAAE: 46753215.5.0000.5259). Todos os participantes assinaram o Termo de Consentimento Livre e Esclarecido (TCLE) antes da sua inclusão no estudo.

O estudo foi observacional transversal, e incluiu indivíduos hipertensos e normotensos de ambos os gêneros, sem distinção de raça, com idade entre 35 e 60 anos e pacientes periodontalmente saudáveis ou com periodontite crônica severa. Os critérios de exclusão foram diagnóstico de diabetes, taxa de filtração glomerular estimada $<60 \mathrm{~mL} / \mathrm{min} / 1,73 \mathrm{~m}[2]$, tabagismo atual ou pregresso (durante o último ano), uso de antibióticos nos útimos 6 meses, indivíduos com diagnóstico de doenças malignas, autoimunes, síndrome da imunodeficiência adquirida, insuficiência cardíaca, doença vascular periférica, doença pulmonar e insuficiência hepática, tratamento odontológico em até 6 meses antes da inclusão no estudo, presença de menos de 6 dentes naturais, pacientes com história de infarto agudo do miocárdio ou acidente vascular encefálico.

Foram selecionados 54 pacientes. Os pacientes hipertensos foram selecionados na Clínica de Hipertensão do CLINEX / UERJ. Os normotensos foram selecionados de outras fontes, incluindo amigos e parentes de pacientes, funcionários do HUPE e da clínica de Periodontia da Faculdade de Odontologia da UERJ. Todos os participantes que preencheram os critérios de elegibilidade foram agendados e receberam as orientações necessárias para comparecer ao Laboratório do CLINEX/UERJ. Essas orientações incluíram jejum de 12 horas e abstinência de álcool por 3 dias para avaliação laboratorial, da composição corporal e da função microvascular.

Entre os 54 pacientes, 5 foram excluídos, 3 por não comparecimento para realização de parte dos exames, mesmo após mais de cinco tentativas de marcação dos exames, 1 por possuir apenas 5 dentes naturais e 1 por ter sido descoberto posteriormente que já havia sofrido infarto agudo do miocárdio.

No laboratório CLINEX/UERJ era feita uma rápida avaliação periodontal, para análise inicial do número de dentes e presença ou ausência de periodontite grave. Em seguida eram submetidos aos exames em jejum, incluindo coleta de sangue para realização dos exames laboratoriais, avaliação da reatividade microvascular cutânea e Bioimpedância elétrica tetrapolar. Após a realização desses exames, os voluntários recebiam um lanche e eram encaminhados para a avaliação periodontal.

\section{Exame clínico periodontal}

O exame periodontal incluiu avaliação do (1)índice de placa (IP), (2) profundidade de bolsa à sondagem (PBS), (3) índice de sangramento à sondagem (SS) (análise dicotômica) e (4) nível de inserção clínica (NIC). PBS, SS e NIC foram avaliados em seis sítios (mesiovestibular, vestibular, distovestibular, mesiolingual, lingual e distolingual) e o IP em quatro sítios (mesial, vestibular, lingual e distal). Todas as medidas foram realizadas 


\section{Artigo original}

através de uma sonda periodontal milimetrada calibrada (Hu-Friedy® PCP15, Chicago, USA) e os valores obtidos aproximados para o milímetro mais próximo. Todos os pacientes foram examinados por um único examinador (RHS).

Os pacientes foram divididos em 4 grupos: G1normotensos sem periodontite ( $\mathrm{n}=11), \mathrm{G} 2$ - hipertensos sem periodontite $(n=16), G 3-$ normotensos com periodontite $(n=9)$ e G4-normotensos com periodontite $(n=13)$. Pacientes diagnosticados com periodontite deveriam apresentar pelo menos 2 sítios em dentes não consecutivos com PBS $\geq 6 \mathrm{~mm}$ e NIC $\geq 6 \mathrm{~mm}$. Foram classificados como hipertensos aqueles voluntários já diagnosticados por um médico e que faziam uso de medicação anti-hipertensiva.

\section{Características demográficas, clínicas e estilo de vida}

Os dados coletados incluíram dados pessoais, endereço, idade, data de nascimento e uso de medicações. Posteriormente, eram feitas 6 aferições da Pressão Arterial (PA), após um prévio período de repouso e ao menos 2 das medições foram feitas com o paciente deitado. A pressão arterial e a frequência cardíaca foram mensuradas por método oscilométrico, com aparelho semiautomático validado, da marca OMRON (IntelliSense® modelo HEM-742INT).

\section{Exames laboratoriais}

Os participantes do estudo coletaram sangue, após 12 horas de jejum, no Laboratório da Disciplina de Fisiopatologia Clínica eExperimental eo processamento do mesmo foi feito atendendo as especificações próprias de cada variável. Alíquotas de plasma e soro foram armazenadas a $-80{ }^{\circ} \mathrm{C}$ para as determinações laboratoriais. Os níveis de colesterol total, colesterol da lipoproteína de alta densidade (HDL-c) e triglicerídios foram determinados pelo método colorimétrico enzimático. O colesterol da lipoproteína de baixa densidade (LDL-c) foi estimado através da fórmula de Friedewald. A creatinina foi determinada pelo método cinético de reação de Jaffé (cobas integra 400 plus). As concentrações séricas de ácido úrico, proteínas totais, globulina e albumina foram determinas pelo método colorimétrico. Os níveis séricos de sódio e potássio foram avaliados pelo método eletrodo seletivo e a de ureia pelo método cinético. A glicemia em jejum foi determinada pelo método enzimático hexoquinase. $\mathrm{O}$ hemograma completo foi analisado por metodologia automatizada, e a hemoglobina glicada através da cromatografia líquida de alta performance.

\section{Avaliação da reatividade microvascular cutânea}

A reatividade microvascular cutânea foi avaliada por meio da técnica de fluxometria laser speckle (LSCI), segundo protocolo estabelecido, padronizado e validado. ${ }^{26-28} \mathrm{O}$ sistema de fluxometria de laser speckle (comprimento de onda de $780 \mathrm{~nm}$; PeriCam PSI-NR, Perimed AB, Järfälla, Suécia) foi utilizado para a avaliação não-invasiva e em tempo real das variações de fluxo microvascular e da hiperemia reativa pós oclusiva (HRPO).

Para avaliação não invasiva e em tempo real da variação de fluxo microvascular usou-se o sistema de fluxometria de laser speckle (comprimento de onda de 785 nm; PeriCam PSI-NR, Perimed AB, Järfälla, Suécia). As imagens foram analisadas pelo software do fabricante (PIMSoft, Perimed, Järfälla, Sweden). Para essa avaliação os pacientes permaneceram 20min. em repouso na posição supina em ambiente com temperatura controlada $(23 \pm 1 \mathrm{oC})$ e após jejum de $12 \mathrm{~h}$. Todos os indivíduos fizeram uso das suas medicações habituais na manhã do teste.

As medidas de fluxo microvascular cutâneo são expressas em Unidades Arbitrárias de Perfusão (UAP) de acordo com o consenso europeu (European Laser Doppler User's Groups, London 1992). O valor máximo em UAP foi registrado. A razão entre os valores máximos em UAP e a pressão arterial média representou a Condutância Vascular Cutânea (CVC), expressa como unidades arbitrárias de perfusão por mmHg (UAP/ $\mathrm{mmHg}$ ). Também foi avaliada a área de fluxo/tempo, também chamada de área abaixo da curva (AUC), método também validado, representativo da variação global de fluxo microvascular em resposta a vários estímulos. ${ }^{29}$

Para a manobra de HRPO foi aplicada por 3 minutos uma oclusão do braço por manguito de esfigmomanômetro a uma pressão $50 \mathrm{mmHg}$ acima da pressão arterial sistólica. Após a liberação do manguito, procedeu-se a medida do fluxo máximo, obtendo-se a CVC da mesma forma descrita anteriormente. A amplitude da resposta foi expressa como a CVC basal subtraída da CVC máxima.

\section{Avaliação antropométrica}

As mensurações de peso corporal (precisão de 0,1 $\mathrm{kg}$ ) e estatura (precisão de $0,5 \mathrm{~cm}$ ) foram realizadas em balança antropométrica digital. Para realização dessas medidas os pacientes estavam em jejum, descalços 
e vestindo roupas leves. O índice de massa corpórea (IMC) foi calculado através da divisão do peso $(\mathrm{kg})$ pela estatura $\left(\mathrm{m}^{2}\right)$. A obesidade foi caracterizada quando o IMC era $\geq 30 \mathrm{~kg} / \mathrm{m}^{2}$.

\section{Avaliação da composição corporal}

A avaliação da composição corporal (determinação da massa magra e da massa gorda) foi realizada por bioimpedância elétrica tetrapolar. A BIA foi realizada utilizando o equipamento Biodynamics modelo $450 \circledR$ (Biodynamics, WA, EUA). Os pacientes foram orientados a jejuar pelo mínimo 4 horas e não consumir alimentos com quantidade significativa de cafeína como café e chás, além de refrigerantes à base de cola e bebidas alcoólicas, no dia anterior ao exame. Além disso, não deviam promover mudanças na intensidade ou frequência dos exercícios físicos nas 48 horas anteriores à realização desta avaliação. Os pacientes foram orientados a esvaziar a bexiga antes do exame. Os eletrodos foram fixados do lado direito do corpo, e os pacientes posicionados deitados sobre uma maca, sem sapatos e meias; livre de qualquer metal em contato com o corpo (bijouterias, joias, cinto, óculos), com os braços separados do corpo num ângulo aproximado de $30^{\circ}$, e as pernas separadas entre si num ângulo de mais ou menos de $40^{\circ} .30,31$ As regiões onde os eletrodos seriam inseridos foram higienizadas com álcool isopropílico 70, e foram posicionados, conectados ao aparelho. Dessa forma, as informações de peso, idade, sexo e altura eram fornecidas. Após média de um minuto, todas as medidas de interesse, em especial, massas magra e gorda, além do ângulo de fase, foram obtidas.

\section{Análise estatística}

Os dados foram inseridos no programa SPSS 23.0
(SPSS, Inc. Chicago, USA). O teste Kolmogorov-Smirnov foi utilizado para verificar a normalidade dos dados avaliados. Os dados de distribuição não normal estão apresentados em medianas (intervalo interquartil), e o teste não-paramétrico de Kruskal Wallis foi utilizado para verificar diferenças significantes nos parâmetros entre os exames dos grupos. Os dados de distribuição normal estão apresentados em médias ( \pm desvio padrão), enquanto o teste de ANOVA foi utilizado para verificar diferenças nesses parâmetros. O teste do qui-quadrado foi utilizado para verificar diferenças nos dados categóricos. Foi considerada a significância estatística para $\mathrm{p}<0,05$.

\section{Resultados}

A tabela 1 mostra a mediana (intervalo interquartil) da idade, raça, sexo, PA sistólica e diastólica e pulso médio e frequência de raça e sexo feminino. Não foram observadas diferenças em relação a idade, distribuição de raça e gênero, e valores médios de PA sistólica e diastólica e nem de pulso médio entre os 4 grupos analisados.

Na tabela 2 são apresentados os dados coletados durante o exame periodontal. Indivíduos do grupo G1 apresentaram valores médios significativamente maiores de número de dentes, quando comparados aos demais grupos. G1 apresenta valores médios de SS menores que os observados nos grupos G2 e G4. Os valores médios de IP eram significativamente maiores no G4, quando comparados aos observados em G1. Os grupos G3 e G4, que compreendem os pacientes sem DP, apresentam valores de $\mathrm{PBS} \geq 4$ e NIC $>6$, significativamente maiores que os grupos G1 e G2.

A tabelas 3 apresenta os resultados dos exames laboratoriais. Não houve diferença significativa entre

Tabela 1. Características da população do estudo

\begin{tabular}{|c|c|c|c|c|c|}
\hline Variáveis & G1 $(n=11)$ & G2 (n=16) & G3 (n=9) & G4 (n=13) & $\mathbf{p}$ \\
\hline Idade & $50(7)$ & $56(8,5)$ & $49(11,5)$ & $53(9,5)$ & NS \\
\hline Raça branca n (\%) & $4(36,4)$ & $5(31,3)$ & $2(22,2)$ & $3(23,1)$ & 0,02 \\
\hline Sexo feminino $\mathrm{n}(\%)$ & $5(45,5)$ & $13(81,3)$ & $6(66,7)$ & $6(46,2)$ & NS \\
\hline PA sistólica & $134(28)$ & $141,5(20,8)$ & $119(32,5)$ & $128(24)$ & NS \\
\hline PA diastólica & $83(18)$ & $79(14,8)$ & $81(20)$ & $82(12)$ & NS \\
\hline Pulso médio & $71(26)$ & $64(9,8)$ & $73(8)$ & 65 (19) & NS \\
\hline
\end{tabular}

Legenda: G1- pacientes normotensos sem periodontite, G2- pacientes hipertensos sem periodontite, G3- pacientes normotensos com periodontite, G4- pacientes hipertensos com periodontite

Teste qui-quadrado foi utilizado para avaliar raça e sexo, e o teste Kruskal Wallis para os demais itens 


\section{Artigo original}

Tabela 2. Avaliação periodontal expressa em mediana (intervalo interquartil)

\begin{tabular}{|c|c|c|c|c|c|}
\hline Variáveis & $\mathrm{G} 1(\mathrm{n}=11)$ & $G 2(n=16)$ & G3 (n=9) & G4 $(n=13)$ & $\mathbf{p}$ \\
\hline No de dentes & $28(1)$ * & 19,5 (12) & $19(10,5)$ & $22(11)$ & 0,01 \\
\hline IP (\%) & $4,5(10,2)$ & $5,3(24,2)$ & $13,4(42,3)$ & $24(19,7) \#$ & 0,03 \\
\hline SS (\%) & $4,8(12,4) ¥$ & $16,4(24,1)$ & $34(43,3)$ & $21,3(26,4)$ & 0,01 \\
\hline PBS 4-5 mm (\%) & $1(2)$ & $2(4)$ & $22(22,5) \alpha$ & $25(20) \alpha$ & 0,000 \\
\hline PBS > $6 \mathrm{~mm}(\%)$ & $0(0)$ & $0(1)$ & $5(4,5) \alpha$ & $8(9) \alpha$ & 0,000 \\
\hline NIC 4-5 mm (\%) & $2(3)$ & $5,5(6)$ & $33(24) \alpha$ & $40(28,5) \alpha$ & 0,000 \\
\hline NIC > 6 mm (\%) & $0(1)$ & $0(1)$ & $10(12,5) \alpha$ & $11(13) \alpha$ & 0,000 \\
\hline
\end{tabular}

Legenda: G1- pacientes normotensos sem periodontite, G2- pacientes hipertensos sem periodontite, G3- pacientes normotensos com periodontite, G4- pacientes hipertensos com periodontite.

* G1 significantemente maior que os valores de G2, G3 e G4.

\# G4 significantemente maior que os valores de G1.

$¥ \mathrm{G} 1$ significantemente menor que os valores de $\mathrm{G} 2$ e $\mathrm{G} 4$.

$\alpha \mathrm{G} 3$ e G4 significantemente maiores que os valores de $\mathrm{G} 1$ e G2.

Tabela 3. Perfil glicêmico, ureia, creatinina e ácido úrico, perfil lipídico, proteínas totais, globulina, albumina, níveis de sódio e potássio através de mediana (intervalo interquartil)

\begin{tabular}{|c|c|c|c|c|c|}
\hline Variáveis & $\mathrm{G} 1(\mathrm{n}=11)$ & G2 $(n=16)$ & G3 (n=9) & G4 (n=13) & p \\
\hline Glicemia & $88(3,5)$ & $95,5(21,8)$ & $96(11,8)$ & $98(14,8)$ & NS \\
\hline Hemoglicada & $5,4(0,7)$ & $5,9(0,7)$ & $5,3(0,8)$ & $5,9(0,8)$ & NS \\
\hline Uréia & $29(10)$ & $32,5(11,5)$ & $25(13)$ & $25(10)$ & NS \\
\hline Creatinina & $0,9(0,3)$ & $0,7(0,2)$ & $0,8(0,4)$ & $0,8(0,4)$ & NS \\
\hline CT (mg/dl) & $209,2(29,3)$ & $207,9(36,9)$ & $197,8(28,5)$ & $190,5(32,4)$ & NS \\
\hline HDL-C (mg/dl) & $51,1(16,6)$ & $60,2(12,8)$ & $57,6(18,7)$ & $52,2(11,5)$ & NS \\
\hline LDL-C (mg/dl) & $128,4(27,8)$ & $123,5(37,8)$ & $112,8(25,7)$ & $112,7(28,7)$ & NS \\
\hline Triglicerídeos (mg/dl) & $149,8(77,5)$ & $121,8(40)$ & $127,4(33,6)$ & $128,6(84,1)$ & NS \\
\hline PTN total (mg/dl) & $7,2(0,8)$ & $7(0,3)$ & $7,1(0,5)$ & $7,2(0,8)$ & NS \\
\hline Albumina (mg/dl) & $4,4(0,5)$ & $4,4(0,4)$ & $4,3(0,4)$ & $4,3(0,4)$ & NS \\
\hline Globulina (mg/l) & $2,3(0,7)$ & $2,6(0,6)$ & $2,7(0,7)$ & $2,9(0,6)$ & NS \\
\hline Sódio & $141(4,3)$ & $141,5(4)$ & $141(4,3)$ & $140(4)$ & NS \\
\hline Potássio & $4,2(0,5)$ & $4,3(0,5)$ & $4,2(0,3)$ & $4,4(0,4)$ & NS \\
\hline
\end{tabular}

Legenda: G1- pacientes normotensos sem periodontite, G2- pacientes hipertensos sem periodontite, G3- pacientes normotensos com periodontite, G4- pacientes hipertensos com periodontite.

os valores da glicemia, hemoglobina glicada, ureia, creatinina, ácido úrico, perfil lipídico, proteínas totais plasmáticas, globulina, albumina, níveis séricos de sódio e potássio.

Não foram observadas diferenças significativas com relação aos valores médios do pico CVC, da amplitude CVC e UAC (tabela 4).

Os dados da mediana do IMC, das massas magra e gorda e ângulo de fase (AF), são descritos na tabela 5. Os grupos 2 e 4, que são constituídos por pacientes 
Tabela 4. Mediana (intervalo interquartil) da reatividade microvascular cutânea

\begin{tabular}{|c|c|c|c|c|c|}
\hline Variáveis & G1 $(n=11)$ & $G 2(n=16)$ & G3 $(n=9)$ & G4 (n=13) & p \\
\hline Pico CVC & $0,7(0,3)$ & $0,8(0,3)$ & $0,8(0,5)$ & $0,7(0,3)$ & 0,8 \\
\hline Amplitude CVC & $0,5(0,2)$ & $0,5(0,3)$ & $0,5(0,3)$ & $0,5(0,2)$ & 0,9 \\
\hline AUC & 2430 (1857) & 2664 (950) & $2758(1562)$ & $2922(849)$ & 0,9 \\
\hline
\end{tabular}

Legenda: G1- pacientes normotensos sem periodontite, G2- pacientes hipertensos sem periodontite, G3- pacientes normotensos com periodontite, G4- pacientes hipertensos com periodontite.

Tabela 5. Mediana (intervalo interquartil) do IMC, massas magra e gorda, e ângulo de fase

\begin{tabular}{lccccc}
\hline \multicolumn{1}{c}{ Variáveis } & G1 (n=11) & G2 (n=16) & G3 (n=9) & G4 (n=13) & p \\
\hline IMC & $24,3(4,4)$ & $30,7(9,8)$ & $24,5(10)$ & $30,6(4,3)$ & NS \\
\hline Massa gorda & $18,7(9,8)$ & $30,8(12,3)^{*}$ & $21,3(15,4)$ & $28,6(5,2)^{*}$ & 0,007 \\
\hline Massa magra & $50,9(21,4)$ & $53,5(12)$ & $46,5(12,9)$ & $51,9(23,8)$ & 0,5 \\
\hline Ângulo de fase & $6,8(1,9)$ & $6,7(1,3)$ & $7(0,8)$ & $7,6(5,7)$ & 0.15 \\
\hline
\end{tabular}

Legenda: G1- pacientes normotensos sem periodontite, G2- pacientes hipertensos sem periodontite, G3- pacientes normotensos com periodontite, G4- pacientes hipertensos com periodontite.

* G2 e G4 significativamente maiores que os valores de G1 e G3.

hipertensos, demonstraram valores significativamente mais elevados de massa gorda quando comparados aos valores obtidos nos grupos 1 e 2 .

\section{Discussão}

A hipótese inicial do estudo indicava uma associação entre a periodontite crônica grave e alterações no exame LSCI. Os resultados do presente estudo não demonstraram influência da periodontite grave nos resultados da reatividade microvascular cutânea. Não existem estudos na literatura para comparação. No entanto, uma possibilidade para que a hipótese não tenha se confirmado foi o fato de que todos os pacientes hipertensos do estudo faziam acompanhamento médico com cardiologista periodicamente e usavam regularmente a medicação anti-hipertensiva recomendada. Assim, mudanças dos valores de reatividade microvascular cutânea talvez possam ocorrer em pacientes hipertensos recém diagnosticados e que ainda não tenham a PA controlada. Outra possibilidade seria o número reduzido de pacientes nos 4 grupos estudados.

No presente estudo a função vascular foi avaliada através da reatividade microvascular cutânea, por meio da técnica não invasiva de LSCI. ${ }^{32}$ A fluxometria laser speckle foi desenvolvida para monitorização da função microvascular da pele, permitindo monitorar as modificações no fluxo sanguíneo cutâneo em tempo real. Foi demonstrado que a técnica LSCI reduz drasticamente a variabilidade das medidas clínicas em comparação com a técnica de FLD, tornando esta técnica uma possível alternativa para facilitar estudos microvasculares na rotina clínica. ${ }^{33,34}$

A HRPO aplicada no estudo constitui um método não farmacológico muito utilizado, que se refere ao aumento agudo no fluxo sanguíneo no antebraço dentro do tecido observado imediatamente após a liberação da oclusão local arterial.19,29,33 Os mecanismos propostos como responsáveis pela hiperemia cutânea pós-oclusão incluem fatores dependentes e independentes do endotélio. Os fatores que provavelmente estão envolvidos são os fatores hiperpolarizantes derivados do endotélio, sugeridos como os principais contribuintes do endotélio na HRPO. O envolvimento dos nervos sensoriais através de reflexo axônico local também tem sido descrito como um importante contribuinte para a HRPO. ${ }^{19,33}$ Portanto, a HRPO não é um teste de função endotelial microvascular, mas sim uma ferramenta para detectar modificações gerais na função microvascular. ${ }^{27}$ Os resultados obtidos nesse estudo não demonstraram associação entre periodontite grave e obesidade. Por outro lado, os pacientes hipertensos apresentaram maiores índices de massa gorda. Não houve alteração na composição de massa magra em nenhum grupo, nem no AF. A associação entre obesidade e periodontite 


\section{Artigo original}

ainda apresenta dados conflitantes e deve ser bem avaliada devido a presença de fatores comuns que podem causar confusão, entre eles idade, sexo, hábitos de higiene oral e estilo de vida. Há na literatura algumas justificativas para a correlação entre obesidade e hipertensão. Estudos indicam que o ganho de peso futuro é significativamente maior em pacientes com hipertensão do que em indivíduos normotensos, portanto, a hipertensão arterial por si só contribuiria para o aumento do risco de obesidade..$^{35} \mathrm{Em}$ relação à microbiologia, mudanças no microbioma bacteriano intestinal, associadas a fatores genéticos e alimentares, podem levar a distúrbios metabólicos que resultam em obesidade, resistência à insulina e hipertensão. ${ }^{35,36}$ Além disso, disfunção endotelial e rigidez arterial são vistas como as manifestações iniciais de disfunção vascular na obesidade e precedem o desenvolvimento de préhipertensão e hipertensão arterial.O aumento da rigidez arterial é observado em pacientes normotensos obesos e que estão predispostos a desenvolver hipertensão. Assim, a hipertensão é mais prevalente em pacientes que possuem maior rigidez arterial. ${ }^{35,38}$

Em uma metaanálise, Chaffee e Weston ${ }^{40}$ observaram relação positiva entre DP e obesidade que seria também fator de risco para perda dentária em adultos em todo o mundo. Pires e colaboradores ${ }^{41}$ concluíram que a obesidade e DP em pacientes obesos elevam o risco de DCV.Foram encontradas correlações positivas entre sobrepeso e obesidade com níveis elevados de lipídios séricos periféricos e indicadores de periodontite. ${ }^{43}$

Em estudo transversal, o aumento do risco de periodontite foi relatado como consequência do aumento do IMC. ${ }^{44} \mathrm{Em}$ um estudo longitudinal de 12 anos, Lee e colaboradores ${ }^{46}$ associaram obesidade ao maior risco de DP, mesmo após ajustes para sexo, idade, renda familiar, área de residência, condição de saúde e fumo. Park e colaboradores ${ }^{48}$ concluíram que indivíduos obesos possuiam menor freqüência diária de escovação dentária e utilizavam menos produtos secundários para higiene bucal. Então, é interessante que pacientes obesos recebam orientação adequada sobre estratégias de promoção de saúde oral. O fato da maioria dos estudos que relacionam obesidade e DP serem transversais, não permite a identificação da obesidade como fator de risco. Por isso, a relação causal da obesidade e periodontite ainda é controversa. Dessa forma, mais estudos são necessários para avaliar a questão da causalidade.

Concluindo, não houve relação entre periodontite e alterações na reatividade microvascular cutânea. Houve um aumento significativo na massa gorda de pacientes hipertensos.

\section{Referências}

1. Temple LK, McLeod RS, Gallinger S, et al. Essays on science and society: Defining disease in the genomics era. Science. 2001;293:807-8.

2. Kolemberg PE. Oral microbial communities: biofilms, interactions, and genetic systems. Ann Rev Microbiol. 2000;54:423-37.

3. Page RC. Oral health status in the United States: Prevalence of inflammatory periodontal diseases. J Dent Edu. 1985;49:354-64.

4. Oliver RC, Brown LJ, Loe H. Periodontal diseases in the United States population. J Periodontol. 1998;69:269-78.

5. Page RC, Offenbacher S, Schroeder HE, et al. Advances in the pathogenesis of periodontitis: Summary of developments, clinical implications and future directions. Periodontol. 2000;1997(14):216-48.

6. Darveau RP, Tanner A, Page RC. The microbial challenge in periodontitis. Periodontol. 2000;1997(14):12-32.

7. Humphrey LL, Fu R, Buckley DI, et al. Periodontal disease and coronary heart disease incidence: a systematic review and meta-analysis. J Gen Int Med. 2008;23(12):2079-2086.

8. Desvarieux M, Demmer RT, Jacobs DR Jr, et al. Periodontal bacteria and hypertension: the oral infections and vascular disease epidemiology study (INVEST). J Hypertens. 2010;28:1413-21.

9. Tsakos G, Sabbah W, Hingorani AD, et al. Is periodontal inflammation associated with raised blood pressure? Evidence from a National US survey. J Hypertens. 2010;28:2386-93.

10. Vidal F, Cordovil I, Figueredo CM, et al. Non-Surgical periodontal treatment reduces cardiovascular risk in refractory hypertensive patients: a pilot study. J Clin Periodontol. 2013;40:681-687.

11. Widlansky M, Gokce N, Keaney JF Jr, et al. The clinical implications of endothelial dysfunction. J Am Coll Cardiol. 2003;42:1149-60.

12. Libby $P$, Theroux P. Pathophysiology of coronary artery disease. Circulation. 2005;111:3481-88.

13. Teles R, Wang C-Y. Mechanisms involved in the association between periodontal diseases and cardiovascular disease. Oral Dis. 2011;17:450-61

14. Lira- Júnior R, Figueredo CM, Bouskela E, et al. Severe chronic periodontitis is associated with endothelial and microvascular dysfunctions: a pilot study. J Periodontol. 2014;85(12):1648-57.

15. Hujoel PP, Drangsholt M, Spiekerman C, et al. Periodontal disease and coronary heart disease risk. JAMA. 2000;284:1406-10.

16. Paizan MLM, Vilela-Martin JF. Is There an Association between Periodontitis and Hypertension? Current Cardiol Rev. 2014;10:355-361.

17. Bahia L, Aguiar LGK, Villela NR, et al. Endotélio e aterosclerose. Revista da SOCERJ. 2004;17(1):26-32.

18. Oliver JJ, Webb DJ. Noninvasive assessment of arterial stiffness and risk of atherosclerotic events. Arterioscler Vasc Biol. 2003;23:554-66.

19. Cracowski JL, Roustit M. Current methods to assess human cutaneous blood flow. An updated focus on laser based-techniques. Microcirculation. 2016;23:337-344.

20. Hujoel PP. Dietary carbohydrates and dental - systemic diseases. J Dent Res. 2009;88: 490-502.

21. Chapple ILC, Bouchard P, Cagetti MG, et al. Interaction of lifestyle, behaviour or systemic diseases with dental caries and periodontal diseases: consensus report of group 2 of the 
Raquel H. de Souza e cols • Periodontite, hipertensão e Reatividade microvascular cutânea

joint EFP/ ORCA workshop on the boundaries between caries and periodontal diseases. J Clin Periodontol. 2017;44(Suppl. 18):S39-S51.

22. Baumgartner RN, Chumlea WC, Roche AF. Bioelectric impedance phase angle and body composition. Am J Clin Nutr. 1988;48(1):16-23.

23. Moynihan P, Petersen PE. Diet, nutrition and the prevention of dental diseases. Public Health Nutrition. 2004;7:201-26.

24. Östberg AL, Nyholm M, Gullberg B, et al. Tooth loss and obesity in a defined Swedish population. Scand J Public Health. 2009;37:427-33.

25. Lutoslawska G, Malara M, Tomaszewski P, et al. Relationship between the percentage of body fat and surrogate indices of fatness in male and female Polish active and sedentary students. J Physiol Anthropol. 2014;33(1):10.

26. Souza EG, De Lorenzo A, Huguenin G, et al. Impairment of systemic microvascular endothelial and smooth muscle function in individuals with early-onset coronary artery disease: studies with laser speckle contrast imaging. Coronary Art Dis. 2014;25(1):23-8.

27. Eickemberg M, Oliveira CC, Roriz AKC, et al. Bioelectric impedance analysis and its use for nutritional assessments. Rev Nutr. 2011;24(6):883-893.

28. Roustit M, Millet C, Blaise S, et al. Excellent reproducibility of laser speckle contrast imaging to assess skin microvascular reactivity. Microvasc Res. 2010;80(3):505-11.

29. Rosseau P, Mahé G, Haj-Yassin F, et al. Increasing the "region of interest" and "time of interest", both reduce the variability of blood flow measurements using laser speckle contrast imaging. Microvasc Res. 2011;82:88-91.

30. Kyle UG, Bosaeus I, De Lorenzo AD, et al. Bioelectrical impedance analysis - part I: review of principles and methods. Clin Nutr. 2004;23:1226-46.

31. Cômodo ARO, Dias ACF, Tomaz BA, et al. Utilização da
Bioimpedância para Avaliação da Massa Corpórea. Autoria: Associação Brasileira de Nutrologia Sociedade Brasileira de Nutrição Parenteral e Enteral. 2009. Disponível em http://www. projetodiretrizes.org.br/8_volume/39- Utilizacao.pdf. Acesso em: 20 de agosto de 17.

32. Briers D, Duncan DD, Hirst E, et al. Laser speckle contrast imaging: theoretical and practical limitations. J Biomed Opt. 2013;18(6):066018.

33. Demarco VG, Aroor AR, Sowers JR. The pathophysiology of hypertension in patients with obesity. Nat Rev Endocrinol. 2014;10(6):364-376.

34. Cavalcante JL, Lima JA, Redheuil A, et al. Aortic stiffness: current understanding and future directions. J Am Coll Cardiol. 2011;57:1511-1522.

35. Chaffee BW, Weston SJ. Association between chronic periodontal disease and obesity: a systematic review and meta-analysis. J Periodontol. 2010;81:1708-1724.

36. Pires JR, Dos Santos IP, de Camargo LF, et al. Framingham cardiovascular risk in patients with obesity and periodontitis. $J$ Indian Soc Periodontol. 2014;18:14-8.

37. Slotwinska SM, Slotwinska R. Host response, obesity, and oral health. Central European J Immunol. 2015;40(2):201-5.

38. Shrestha S, Shrestha RM. Correlation between oral health and body mass index among Nepalese teachers. Kathmandu Univ Med J (KUMJ). 2016;14(55):231-34.

39. Lee JH, Oh JY, Youk TM, et al. Association between periodontal disease and non-communicable diseases. 12-year longitudinal health-examinee cohort study in South Korea. Medicine. 2017;96:26.

40. Park J-B, Nam GE, Han K, et al. Obesity in relation to oral health behaviors: An analysis of the Korea National Health and Nutrition Examination Survey 2008-2010. Exp Therap Med. 2016;12:3093-3100. 